\title{
ANÁLISIS DE COSTOS DE LOS MÉTODOS RÁPIDOS PARA DIAGNÓSTICO DE TUBERCULOSIS MULTIDROGORRESISTENTE EN DIFERENTES GRUPOS EPIDEMIOLÓGICOS DEL PERÚ
}

\author{
Lely Solari1,a, Alfonso Gutiérrez-Aguado ${ }^{1, a}$, Carmen Suárez ${ }^{1, b}$, Oswaldo Jave ${ }^{2, a}$, Edith Castillo 3 ,b, \\ Gloria Yale ${ }^{4, a}$, Luis Ascencios ${ }^{1, b}$, Neyda Quispe ${ }^{1, b}$, Eddy Valencia ${ }^{1, b}$, Víctor Suárez $^{1, a}$
}

\begin{abstract}
RESUMEN
Objetivos. Evaluar los costos de tres métodos diagnósticos para susceptibilidad a drogas antituberculosas y comparar el costo por caso de tuberculosis multidrogorresistente (TB MDR) diagnosticado con estos (MODS; GRIESS y Genotype MTBDR plus $®$ ) en cuatro grupos epidemiológicos en el Perú. Materiales y métodos. En base a cifras programáticas, se dividió a la población en cuatro grupos: pacientes nuevos de Lima/Callao; nuevos de otras provincias; los antes tratados de Lima/Callao y de otras provincias. Se calcularon los costos de cada prueba en base a la metodología estándar utilizada por el Ministerio de Salud, desde la perspectiva de los servicios de salud. Basado en ello, se calculó el costo por paciente TB MDR diagnosticado para cada grupo epidemiológico. Resultados. Los costos estimados por prueba para MODS, GRIESS, y Genotype MTBDR plus ${ }^{\circledR}$ fueron de 14,83; 15,51 y 176,41 nuevos soles, respectivamente. El costo por paciente TB MDR diagnosticado con GRIESS y MODS fue menor a los 200 nuevos soles en tres de los cuatro grupos. El costo por TB MDR diagnosticado fue de más de 2000 nuevos soles con el Genotype MTBDR plus $®$ en los dos grupos de pacientes nuevos y, menores a 1000 nuevos soles en los grupos de pacientes antes tratados. Conclusiones. En grupos de alta prevalencia, como son los pacientes antes tratados, los costos por caso diagnosticado de TB MDR con las tres pruebas evaluadas fueron bajos, sin embargo, con la prueba molecular en los grupos de baja prevalencia, fueron elevados. El uso de las pruebas moleculares debe optimizarse en grupos de altas prevalencias.
\end{abstract}

Palabras clave: Diagnóstico; Tuberculosis fármaco-resistente; Economía de la salud; Perú (fuente: DeCS BIREME).

\section{COST ANALYSIS OF RAPID METHODS FOR DIAGNOSIS OF MULTIDRUG RESISTANT TUBERCULOSIS IN DIFFERENT EPIDEMIOLOGIC GROUPS IN PERU}

\begin{abstract}
Objectives. To evaluate the costs of three methods for the diagnosis of drug susceptibility in tuberculosis, and to compare the cost per case of Multidrug-resistant tuberculosis (MDR TB) diagnosed with these (MODS, GRIESS and Genotype MTBDR plus $(\mathbb{B})$ in 4 epidemiologic groups in Peru. Materials and methods.In the basis of programmatic figures, we divided the population in 4 groups: new cases from Lima/Callao, new cases from other provinces, previously treated patients from Lima/Callao and previously treated from other provinces. We calculated the costs of each test with the standard methodology of the Ministry of Health, from the perspective of the health system. Finally, we calculated the cost per patient diagnosed with MDR TB for each epidemiologic group. Results. The estimated costs per test for MODS, GRIESS, and Genotype MTBDR plus ${ }^{\circledR}$ were 14.83. 15.51 and 176.41 nuevos soles respectively (the local currency, 1 nuevos sol=0.36 US dollars for August, 2011). The cost per patient diagnosed with GRIESS and MODS was lower than 200 nuevos soles in 3 out of the 4 groups. The costs per diagnosed MDR TB were higher than 2,000 nuevos soles with Genotype MTBDR plus $\AA$ in the two groups of new patients, and lower than 1,000 nuevos soles in the group of previously treated patients. Conclusions. In high-prevalence groups, like the previously treated patients, the costs per diagnosis of MDR TB with the 3 evaluated tests were low, nevertheless, the costs with the molecular test in the low-prevalence groups were high. The use of the molecular tests must be optimized in high prevalence areas.
\end{abstract}

Key words: Diagnosis; Tuberculosis, multidrug-resistant; Health ecomomics; Peru (source: MeSH NLM).

Instituto Nacional de Salud del Perú. Lima, Perú.

Estrategia Sanitaria Nacional de Tuberculosis, Ministerio de Salud. Lima, Perú.

Laboratorio Referencial DIRESA Callao. Callao, Perú.

Laboratorio Referencial DISA Lima-Ciudad. Lima, Perú.

Médico; ' Biólogo

Recibido: 03-06-11 Aprobado: 10-08-11 


\section{INTRODUCCIÓN}

La tuberculosis multidrogorresistente (TB MDR), definida como tuberculosis resistente a isoniazida y rifampicina, es un problema de salud pública de creciente importancia en el Perú. Según el último estudio de prevalencia de resistencia a drogas antituberculosas, la prevalencia global en Perú es del 5,3\% pero varía de manera importante de acuerdo con el estado de tratamiento (pacientes "nuevos" frente a "antes tratados") y a la localización geográfica (pacientes viviendo en Lima-Callao frente a el resto de provincias), dando como resultado de estas combinaciones cuatro grupos epidemiológicos con distinta prevalencia de TB MDR (1).

Un componente crucial de los programas de control de TB MDR es el diagnóstico temprano de la resistencia a drogas y el acceso oportuno a las drogas de segunda línea. Las pruebas microbiológicas "convencionales", como el método de proporciones en Lowenstein-Jenssen, aunque son consideradas el "estándar de referencia", demoran por lo menos tres meses en dar resultados de susceptibilidad a drogas antituberculosas, por lo cual su utilidad clínica es limitada(2). En respuesta a ello, se han desarrollado diversas pruebas consideradas "rápidas" para el diagnóstico de esta condición y que se distinguen en fenotípicas y genotípicas (moleculares).

En la Red Nacional de Laboratorios en Salud Pública del Perú, se vienen utilizando tres de ellas: el método de nitrato reductasa (Griess) que demora alrededor de 1421 días en dar resultados ${ }^{(3)}$, el método de observación microscópica de la susceptibilidad a drogas (MODS), que demora un promedio de siete a catorce días ${ }^{(4)} y$ el método molecular de identificación de mutaciones asociadas con la resistencia a isoniazida y rifampicina (Genotype MTBDR plus ${ }^{\circledR}$ ), que solo requiere de horas para obtener resultados ${ }^{(5)}$.

Si bien estos tres métodos, de acuerdo con la literatura médica, tienen una exactitud diagnóstica bastante elevada para TB MDR ${ }^{(6,7)}$, hay algunas características que los hacen diferentes entre sí. Estas son básicamente referidas a consideraciones de implementación, como los requerimientos de facilidades de laboratorio, el nivel de entrenamiento de personal necesario $y$, sobre todo, económicas, puesto que los costos de estos métodos varían significativamente. En salud pública, al escoger una prueba de laboratorio para el diagnóstico de una condición en particular, uno debe tener en cuenta todas estas consideraciones, así como la prevalencia de la condición en la zona, antes de decidir el método a implementar en los distintos laboratorios a nivel nacional ${ }^{(8)}$.
El presente estudio tiene como objetivo evaluar los costos de cada uno de estos tres métodos diagnósticos, en la Red Nacional de Laboratorios en Salud Pública del Perú, y utilizar simulaciones basadas en cifras programáticas para comparar el costo por caso de TB MDR diagnosticado usando estos métodos en los distintos grupos de prevalencia de TB MDR existentes en el país.

\section{MATERIALES Y MÉTODOS}

El presente estudio comprende dos partes: la primera es una evaluación económica parcial (análisis de costos) y la segunda es una evaluación de costoefectividad desde la perspectiva del financiador. Fue realizado en la Unidad de Análisis y Generación de Evidencias en Salud Pública del Instituto Nacional de Salud (UNAGESP-INS).

\section{MÉTODOS DE LABORATORIO}

Los tres métodos de laboratorio a ser comparados son:

Griess: método basado en la determinación de la actividad de la nitrato reductasa en cultivos de crecimiento activo del M. tuberculosis que da como resultado una reacción colorimétrica, este cambio de color permite la detección temprana del crecimiento de M. tuberculosis. Se comparan tubos controles sin fármacos con tubos que contienen fármacos antituberculosos. Actualmente está siendo utilizado en los laboratorios referenciales de las direcciones de salud (DISA) Lima Este y Lima Ciudad. Para la determinación de la sensibilidad para el diagnóstico de TB MDR, se utilizó la única publicación encontrada en MEDLINE que proporciona esta información (9), la cual reporta una sensibilidad de $97 \%$ (todas las otras publicaciones sobre este método reportan de forma separada sensibilidad a isoniazida y rifampicina).

- MODS: método basado en el crecimiento del $M$. tuberculosis (MTB) en placas conteniendo muestras de esputo descontaminadas y resuspendidas en medio líquido (caldo Middlebrook 7H9 suplementado) y evalúa su susceptibilidad frente a isoniazida (INH) y rifampicina (RIF) mediante la detección con un microscopio de luz invertida del crecimiento de microcolonias en forma de cordón, a partir del quinto día. Actualmente está siendo utilizado en los laboratorios referenciales de las DISA Lima Sur, Arequipa y la DIRESA Callao. La sensibilidad para MDR se extrajo de una publicación basada en pacientes peruanos, la cual se seleccionó porque tiene un diseño de estudio adecuado y un tamaño muestral considerable; esta publicación reporta una sensibilidad para MDR de $91,2 \%{ }^{(4)}$. 
- Genotype MTBDR plus ®: método basado en la tecnología DNA STRIP y permite la identificación mediante genética molecular del complejo $M$. tuberculosis y la resistencia a rifampicina o isoniazida, de muestras de cultivo o esputo. La identificación de la resistencia a rifampicina se basa en la detección de las mutaciones más significativas del gen rpoB. Para detectar la resistencia de isoniazida de alto nivel es examinado el gen katG (que codifica para la catalasa peroxidasa), y para la resistencia de isoniazida de bajo nivel, es examinada la región del promotor del gen inhA (que codifica por la NADH enoil ACP reductasa). Actualmente, está siendo usado en el Laboratorio Nacional de Mycobacterias del INS. La sensibilidad para MDR se encontró en tres publicaciones ${ }^{(5,10,11)}$; se decidió emplear la sensibilidad reportada $(97,1 \%)$ en la publicación rusa realizada en muestras frescas y no congeladas de esputo, y que mostraba lo que ocurre en condiciones operacionales ${ }^{(11)}$.

\section{CÁLCULO DE LOS COSTOS}

Los costos fueron calculados desde la perspectiva del financiador de acuerdo con la "Metodología para la estimación de costos estándar en los establecimientos de salud", que es la guía utilizada por el Ministerio de Salud del Perú (MINSA) para definir los costos en el plan esencial de Aseguramiento en Salud (PEAS), Seguro Integral, etc. ${ }^{(12) .}$ Los costos de los insumos de laboratorio y de los equipos requeridos para las pruebas fueron extraídos del Sistema Integrado de Gestión Administrativa (SIGA) (un sistema informático donde se cotizan los productos de acuerdo con las compras que vienen siendo realizadas en el sector salud, previo estudio de mercado) del INS actualizado a mayo de 2011, y de las DISA; en caso de no contar con los costos del SIGA, se obtuvo cotizaciones de los proveedores del INS y de las DISA. Los procesos y tiempos de ejecución fueron definidos por personal profesional entrenado en las respectivas técnicas (DIRESA Callao para MODS, DISA Lima Ciudad para Griess, INS para Genotype MTBDR plus $\left.{ }^{\circledR}\right)$. La depreciación de los equipos se calculó de acuerdo a los minutos utilizados por prueba y el tiempo de vida útil del equipo.

Para el cálculo de los salarios del personal de laboratorio, se tomó como referencia los salarios del personal contratado mediante la modalidad de Contrato por Administración de Servicios (CAS), al ser la modalidad más frecuente de relación laboral en este medio. En el caso de MODS y Griess, se tomaron en cuenta los salarios de las direcciones regionales de salud y, en el caso del Genotype MTBDR plus ${ }^{\circledR}$, los salarios del INS.

Para este análisis no se ha incluido los costos de presión de aire negativa recomendados para la elaboración de estas pruebas, ni los costos asociados al transporte.

\section{CÁLCULO DE LA EFECTIVIDAD: NÚMERO DE PACIENTES CON TB MDR DIAGNOSTICADOS}

La prevalencia de TB MDR se obtuvo para cada uno de los cuatro grupos del estudio de prevalencia de resistencia a drogas antituberculosas (pacientes nuevos de Lima, prevalencia de $8,1 \%$, pacientes nuevos de provincias, prevalencia de $2,1 \%$, pacientes antes tratados de Lima, prevalencia de $26,0 \%$ y pacientes antes tratados de provincias, prevalencia de $21,5 \%)^{(1)}$. Para cada uno de los cuatro grupos, se calculó el número de pruebas que se deben realizar por año en base al número de pacientes con tuberculosis pulmonar con baciloscopía positiva en el 2009 consignado en el informe operacional (17 391 nuevos y 2736 antes tratados), distribuidos entre Lima/Callao y resto de provincias (59 \% de pacientes en Lima/Callao). Luego se calculó el número de pacientes con TB MDR diagnosticados con cada una de las pruebas, de acuerdo con el número de TB MDR en cada grupo multiplicado por la sensibilidad de cada una de las pruebas.

\section{CÁLCULO DE LA COSTO-EFECTIVIDAD: COSTO POR PACIENTE MDR DIAGNOSTICADO}

En base al costo obtenido para cada una de las pruebas y el número de pacientes diagnosticados con TB MDR en cada grupo, se calculó el costo por paciente MDR diagnosticado para cada una de las tres pruebas evaluadas.

Por haberse realizado el estudio basado en un análisis de los datos operacionales, no fue sometido a revisión por comités de ética.

\section{RESULTADOS}

\section{COSTOS DE LAS PRUEBAS}

Con base en los datos proporcionados por el Laboratorio de Mycobacterias del INS, la DIRESA Callao y la DISA Lima Ciudad, los costos unitarios para cada una de las pruebas se muestran en la Tabla 1 , siendo el costo estimado en soles de 15,05 para Griess, 14,83 para MODS y 176,41 para Genotype MTBDR plus ${ }^{\circledR}$.

Tabla 1. Estimación del costo de cada prueba en nuevos soles, 2011.

\begin{tabular}{lcrc}
\hline ITEM & Griess & MODS & Genotype \\
\hline Recursos humanos & 4,23 & 3,55 & 15,12 \\
Equipos & 7,17 & 5,75 & 14,91 \\
Infraestructura & 1,09 & 0,91 & 3,86 \\
Insumos & 3,02 & 4,63 & 142,52 \\
TOTAL & 15,51 & 14,83 & 176,41 \\
\hline
\end{tabular}


Tabla 2. Número de pruebas de sensibilidad y pacientes con TB MDR diagnosticados en cada grupo epidemiológico.

\begin{tabular}{lccccc}
\hline \multicolumn{1}{c}{ ÍTEM } & $\begin{array}{c}\text { GRUPO 1 } \\
\text { Nuevos } \\
\text { (Lima/Callao) }\end{array}$ & $\begin{array}{c}\text { GRUPO 2 } \\
\text { Nuevos } \\
\text { (Provincias) }\end{array}$ & $\begin{array}{c}\text { GRUPO 3 } \\
\text { Antes tratados } \\
\text { (Lima/Callao) }\end{array}$ & $\begin{array}{c}\text { GRUPO 4 } \\
\text { Antes tratados } \\
\text { (Provincias) }\end{array}$ & $\begin{array}{c}\text { TOTAL } \\
\text { Prevalencia MDR }\end{array}$ \\
$\begin{array}{l}\text { Número estimado de pruebas de } \\
\text { susceptibilidad (PS) que deberían } \\
\text { hacerse }\end{array}$ & 10261 & 2,1 & 26 & 21,5 & 5,3 \\
$\begin{array}{l}\text { Número estimado de pacientes con } \\
\text { TB MDR con PS / año }\end{array}$ & 831 & 7130 & 1614 & 1122 & 20127 \\
\hline
\end{tabular}

Costo por paciente MDR diagnosticado.

El detalle de costos se encuentra en el Anexo 1.

\section{PRUEBAS A SER REALIZADAS EN LOS CUATRO GRUPOS EPIDEMIOLÓGICOS}

La Tabla 2 muestra la estimación, para cada uno de los grupos planteados, del número de pruebas de susceptibilidad a ser realizadas y del número estimado de pacientes con TB MDR en base a la prevalencia encontrada en el estudio de vigilancia de la resistencia a medicamentos antituberculosos.

\section{COSTO POR PACIENTE MDR DIAGNOSTICADO}

En base a la sensibilidad de cada prueba, se calculó el número de pacientes con TB MDR diagnosticados con cada metodología. Asimismo, en base al número de pruebas requeridas, la Tabla 3 muestra la estimación del costo que demandaría utilizar cada una de estas pruebas en todos los pacientes con baciloscopía positiva del país, estratificada de acuerdo con el grupo epidemiológico.

La Figura 1 muestra el costo por paciente con TB MDR diagnosticado en cada uno de los grupos y para cada una de las tres pruebas evaluadas.

\section{DISCUSIÓN}

En respuesta al serio problema de salud que implica la resistencia a las drogas antituberculosas, en la actualidad están saliendo al mercado cada vez más métodos para diagnosticar esta condición. Existen más de ocho grupos de métodos fenotípicos disponibles y varias empresas han puesto en el mercado métodos genotípicos, entre los cuales destacan el Genotype® y más recientemente el GenXpert ${ }^{(13)}$.

Existen muchos estudios primarios que muestran exactitudes diagnósticas muy elevadas de los métodos fenotípicos como los genotípicos, incluso algunos metanálisis reportan sensibilidades y especificidades mayores a $95 \%$ tanto para isoniazida como para rifampicina, en métodos como el Griess ${ }^{(14)}$, MODS ${ }^{(15)}$ y Genotype MTBDR plus ${ }^{\circledR}{ }^{(5)}$; sin embargo, no solo se debe tomar en cuenta la sensibilidad y especificidad de una prueba diagnóstica al momento de decidir su implementación. Otros factores como la prevalencia de la enfermedad y, por ende, los valores predictivos de las pruebas, la facilidad y velocidad de la transmisión de la resistencia en la comunidad, el tiempo de transmisión, la letalidad o discapacidad por tiempo de demora, los costos y la factibilidad de su implementación, son igualmente importantes ${ }^{(8)}$. El presente estudio se concentra en las consideraciones de costos.

Así, en prevalencias de TB MDR menores de $3 \%$, como las que ocurren en pacientes nuevos de provincias, se observa que el uso del método molecular Genotype MTBDR plus $®$, implica costos mayores a los 8000 soles por paciente diagnosticado de TB MDR. Además, se debe tomar en cuenta, en este tipo de poblaciones con prevalencias bajas, el problema de los falsos positivos, que deben tratar de minimizarse pues tienen una implicancia enorme por el costo de un tratamiento mal indicado. Podría ser una aproximación más adecuada el uso de pruebas fenotípicas como Griess y MODS en estos

Tabla 3. Costos totales de aplicación de cada una de las pruebas en los diferentes grupos en nuevos soles.

\begin{tabular}{lccccr}
\hline \multicolumn{1}{c}{ ITEM } & $\begin{array}{c}\text { GRUPO 1: } \\
\text { Nuevos } \\
\text { (Lima/Callao) }\end{array}$ & $\begin{array}{c}\text { GRUPO 2: } \\
\text { Nuevos } \\
\text { (Provincias) }\end{array}$ & $\begin{array}{c}\text { GRUPO 3: } \\
\text { Antes tratados } \\
\text { (Lima/Callao) }\end{array}$ & $\begin{array}{c}\text { GRUPO 4: } \\
\text { Antes tratados } \\
\text { (Provincias) }\end{array}$ & \multicolumn{1}{c}{ TOTAL } \\
\hline Costo con GRIESS & 159143 & 110591 & 25037 & 17398 & 312170 \\
Costo con MODS & 152166 & 105742 & 23939 & 16636 & 298483 \\
Costo con Genotype & 1810088 & 1257858 & 284768 & 197890 & 3550604 \\
\hline
\end{tabular}




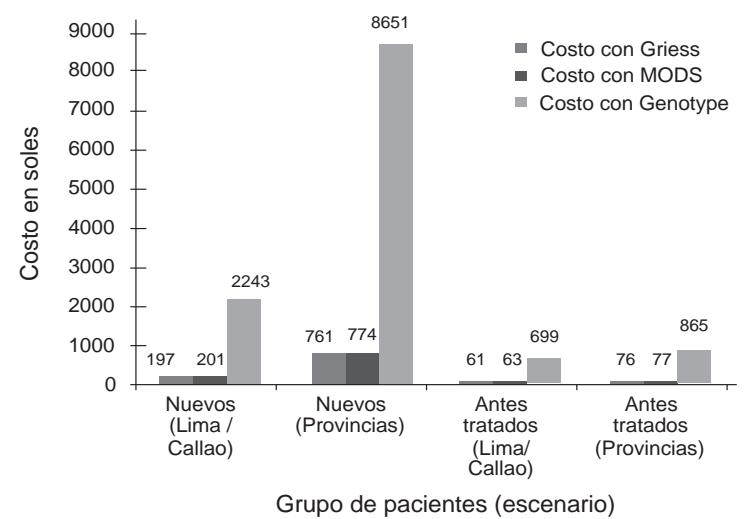

Figura 1. Costo por paciente TB MDR diagnosticado de acuerdo a los grupos utilizando Griess, MODS Y Genotype MTBDR plus $\AA$ en nuevos soles.

grupos, dependiendo de las facilidades de laboratorio y la proporción de casos con baciloscopía negativa, puesto que si es muy alta, MODS podría ser una mejor opción ${ }^{(4)}$, siempre teniendo especial cuidado con no generar sobrediagnóstico. En este sentido, se debe continuar trabajando para que todos los laboratorios referenciales de la Red Nacional de Laboratorios implementen y puedan realizar rutinariamente estas pruebas.

En el caso de pacientes nuevos de Lima y Callao, podemos apreciar que la aplicación de las pruebas moleculares en esta población puede considerarse controversial. Se debe tomar en cuenta que 2000 nuevos soles por paciente TB MDR diagnosticado es una cifra importante. Otra consideración a tomar en cuenta es que dentro de Lima existen distritos con altas prevalencias de TB MDR, las llamadas áreas de elevado riesgo de transmisión de tuberculosis (AERTS), donde, por las altas tasas de resistencia, posiblemente los costos por paciente TB MDR diagnosticado se asemejen a los de los pacientes antes tratados y, en estos, podría ser conveniente aplicar las pruebas moleculares.

No ocurre lo mismo en los grupos 3 y 4 , de pacientes antes tratados en Lima/Callao y provincias. En estos casos, prevalencias de TB MDR por encima de $20 \%$ disminuyen significativamente el costo por paciente MDR diagnosticado a menos de 1000 soles, constituyéndose, desde nuestro punto de vista, en una opción viable y razonable.

Si bien es cierto que no existe un punto de corte claramente definido en cuanto a costos por encima del cual uno pueda respaldar o desaconsejar el uso de una de estas pruebas, es conocido que opciones más económicas son en general más sostenibles por los sistemas de salud a largo plazo ${ }^{(16)}$ y lo más adecuado es optimizar el uso de las pruebas más caras en los grupos que más las requieren, siempre tomando en cuenta otras consideraciones como la oportunidad de diagnóstico temprano, etc.
Este es el primer estudio de análisis de costos de pruebas para diagnóstico de susceptibilidad a drogas antituberculosas en nuestro medio que encuentra resultados similares a los publicados en otros lugares ${ }^{(9)}$. Cabe mencionar ciertas limitaciones del presente estudio; en primer lugar, existen supuestos que no se han considerado y tienen implicancias importantes. Uno de los más relevantes es el referente a los porcentajes de prevalencia en los cuatro grupos epidemiológicos estudiados, puesto que el estudio inicial de vigilancia fue diseñado para calcular una prevalencia de MDR nacional y, en el reporte, es que se realiza la estratificación. Asimismo, las prevalencias de TB MDR en pacientes antes tratados son solo referenciales, puesto que el cálculo del tamaño muestral se realiza con los casos nuevos. Además, el estudio se realizó hace cinco años y la prevalencia de resistencia es probable que haya cambiado. A pesar de estas consideraciones, creemos que los estimados obtenidos en el presente estudio son las mejores aproximaciones de la realidad con la poca información disponible.

En ninguno de los tres casos hemos considerado el costo del entrenamiento del personal, debido a que esto solo se realiza una vez. En el caso de Genotype MTBDR plus $®$, estamos considerando los costos de realizar el mayor número muestras posibles por corrida, que es 45 , y es el escenario ideal, pero no siempre se da.

Un parámetro importante que no ha sido considerado es el tiempo hasta la obtención de resultados, que según la literatura es en promedio de cuatro horas para Genotype MTBDR plus ${ }^{\circledR}$; 7-14 días para MODS y 14-21 días para Griess. Este aspecto es determinante, puesto que mientras más días transcurran hasta el diagnóstico de TB MDR y el inicio del tratamiento respectivo, la posibilidad de progresión de la enfermedad y transmisión a otras personas son mayores. Un análisis formal de costo utilidad que nuestra unidad está llevando a cabo podrá dar respuesta este aspecto.

Otra consideración a tomar en cuenta es que en este estudio solo estamos evaluando el diagnóstico de TBMDR y no estamos realizando el análisis ni para la presencia de mono-resistencia por isoniazida o rifampicina ni para el diagnóstico de XDR, que también es de suma importancia y se realizará en un segundo momento. Asimismo, la sensibilidad y especificidad consideradas para cada uno de los métodos podrían variar de acuerdo con el diseño del estudio en el que fueron evaluadas. Por último, no se realizó un análisis de sensibilidad para ver mejor o peor escenario de costos.

Existen iniciativas, como algunas impulsadas por la OMS, para disminuir los costos de las pruebas moleculares de manera significativa, de concretarse, como es 
esperable a lo largo de los años, e incluso por la aparición de competencia en el mercado, las conclusiones del presente estudio podrían variar.

En conclusión, se aprecia que los costos por paciente diagnosticado de TB MDR con diferentes pruebas para diagnóstico, varían de manera importante con la prevalencia de esta condición. En el Perú, tenemos distintos escenarios de acuerdo a si se trata de pacientes nuevos o antes tratados y, de acuerdo a si viven en Lima /Callao o provincias. Una alternativa razonable podría ser usar pruebas fenotípicas como Griess o MODS en pacientes nuevos en provincias, y pruebas genotípicas o moleculares en pacientes antes tratados o poblaciones con prevalencias similares a las de los pacientes antes tratados (como podrían ser algunos "bolsones" de tuberculosis en distritos de Lima y algunos hospitales). Sin embargo, idealmente estudios formales de costo utilidad deben ser realizados para complementar esta información.

\section{Contribuciones de autoría}

LS participó en la concepción y diseño del trabajo, análisis e interpretación de datos y redacción de la primera versión del manuscrito. AG participó en la concepción y diseño del trabajo, recolección de datos, y análisis e interpretación de datos. CS, OJ y VS participaron en la concepción y diseño del trabajo, y en el análisis e interpretación de datos. EC, GY, LA, NQ, EV participaron en la recolección de datos, análisis e interpretación de datos y redacción de la primera versión del manuscrito. Todos los autores revisaron en forma crítica versiones preliminares del manuscrito y aprobaron la versión final del trabajo.

\section{Fuentes de financiamiento}

Instituto Nacional de Salud.

\section{Conflictos de interés}

Carmen Suarez, Gloria Yale, Luis Ascencios y Neyda Quispe han sido miembro del equipo de investigación de Griess con Socios en Salud. Oswaldo Jave es Jefe de la Estrategia Sanitaria Nacional de Prevención y Control de Tuberculosis. Edith Castillo trabajó como personal contratado por la Universidad Peruana Cayetano Heredia para un estudio de validación del MODS.

\section{REFERENCIAS BIBLIOGRÁFICAS}

1. Asencios L, Quispe N, Mendoza-Ticona A, Leo E, Vásquez L, Jave O, et al. Vigilancia Nacional de la resistencia a medicamentos antituberculosis, Perú 2005-2006. Rev Peru Med Exp Salud Publica. 2009;26(3):278-87.

2. Balabanova $Y$, Drobniewski F, Nikolayevskyy V, Kruuner A, Malomanova N, Simak T, et al. An integrated approach to rapid diagnosis of tuberculosis and multidrug resistance using liquid culture and molecular methods in Russia. PLoS One. 2009;4(9):e7129.
3. Asencios L, Yale G, Yagui M, Quispe N, Taylor A, Blaya $\mathrm{J}$, et al. Programmatic implementation of rapid DST for Mycobacterium tuberculosis in Peru. Int $\mathrm{J}$ Tuberc Lung Dis. 2008;12(7):743-9.

4. Moore DA, Evans CA, Gilman RH, Caviedes L, Coronel J, Vivar A, et al. Microscopic-observation drug-susceptibility assay for the diagnosis of TB. N Engl J Med. 2006;355(15):153950.

5. Barnard M, Albert H, Coetzee G, O'Brien R, Bosman ME. Rapid molecular screening for multidrug-resistant tuberculosis in a high-volume public health laboratory in South Africa. Am J Respir Crit Care Med. 2008;177(7):787-92.

6. Bwanga F, Hoffner S, Haile M, Joloba ML. Direct susceptibility testing for multi drug resistant tuberculosis: a meta-analysis. BMC Infect Dis. 2009;9:67.1

7. Minion J, Leung E, Menzies D, Pai M. Microscopic-observation drug susceptibility and thin layer agar assays for the detection of drug resistant tuberculosis: a systematic review and meta-analysis. Lancet Infect Dis. 2010;10(10):688-98.

8. Van Deun A, Martin A, Palomino JC. Diagnosis of drug-resistant tuberculosis: reliability and rapidity of detection. Int J Tuberc Lung Dis. 2010;14(2):131-40.

9. Bwanga F, Haile M, Joloba ML, Ochom E, Hoffner S. Direct Nitrate Reductase Assay versus Microscopic Observation Drug Susceptibility Test for Rapid Detection of MDR-TB in Uganda. PLoS One. 2011;6(5):e19565.

10. Anek-Vorapong R, Sinthuwattanawibool C, Podewils LJ, McCarthy K, Ngamlert K, Promsarin B, et al. Validation of the GenoTypeMTBDRplus assay for detection of MDR-TB in a public health laboratory in Thailand. BMC Infect Dis. 2010;10:123.

11. Nikolayevskyy V, Balabanova Y, Simak T, Malomanova N, Fedorin I, Drobniewski F. Performance of the Genotype MTBDRPlus assay in the diagnosis of tuberculosis and drug resistance in Samara, Russian Federation. BMC Clin Pathol. 2009;9:2.

12. Ministerio de Salud del Perú. Metodología estándar para la estimación de costos estándar en los establecimientos de Salud [Documento Técnico]. Lima: MINSA; 2008.

13. Boehme CC, Nabeta P, Hillemann D, Nicol MP, Shenai S, Krapp F, et al. Rapid molecular detection of tuberculosis and rifampin resistance. N Engl J Med. 2010;363(11):1005-15.

14. Affolabi D, Odoun M, Sanoussi N, Martin A, Palomino JC, Kestens $\mathrm{L}$, et al. Rapid and inexpensive detection of multidrugresistant Mycobacterium tuberculosis with the nitrate reductase assay using liquid medium and direct application to sputum samples. J Clin Microbiol. 2008;46(10):3243-5.

15. Shiferaw G, Woldeamanuel Y, Gebeyehu M, Girmachew F, Demessie D, Lemma E et al. Evaluation of microscopic observation drug susceptibility assay for detection of multidrug-resistant Mycobacterium tuberculosis. J Clin Microbiol. 2007;45(4):1093-7.

16. Meltzer MI. Introduction to health economics for physicians. Lancet. 2001;358:993-8.

Correspondencia: Lely Solari Zerpa

Dirección: Instituto Nacional de Salud

Capac Yupanqui 1400 - Jesus María, Lima 11, Perú.

Teléfono: (511) 617-6200 anexo: 2109

Correo electrónico: lelysol@hotmail.com 